\title{
Embedding theorems for Lipschitz and Lorentz spaces on lower Ahlfors regular sets
}

\author{
by \\ BARTŁomiej Dyda (Wrocław)
}

\begin{abstract}
We prove norm inequalities between Lorentz and Besov-Lipschitz spaces of fractional smoothness.
\end{abstract}

1. Main results. In what follows we let $(F, \rho)$ be a metric space with a positive $\sigma$-finite Borel measure $\mu$. By $B(x, r)$ we denote the open ball centred at $x$ with radius $r$. We always assume that there exist $d>0$ and $C_{1}>0$ such that

$$
\mu(B(x, r)) \geq C_{1} r^{d} \quad \text { for all } 0<r \leq 1 \text { and } x \in F,
$$

i.e., the lower Ahlfors $d$-regularity of $F$. In particular, $F$ may be a $d$-set in $\mathbb{R}^{n}$ and $\mu$ the $d$-dimensional Hausdorff measure, or $F$ may be an $h$-set with $h(r) \geq r^{d}$ for $0<r \leq 1$ and $\mu$ an $h$-measure [10, 11, 5, 6, 18].

We denote $L^{p}=L^{p}(F, \mu)$. We obtain the following inequality of Sobolev type.

Theorem 1. If $0<p<\infty$ and $0<\alpha<d / p$, then there exists $a$ constant $c=c\left(d, C_{1}, p, \alpha\right)$ such that

$$
\begin{aligned}
& \|u\|_{L^{p d /(d-\alpha p)}} \\
& \leq c\left(\|u\|_{L^{p}}+\left(\iint_{\rho(x, y)<1} \frac{|u(x)-u(y)|^{p}}{\rho(x, y)^{d+\alpha p}} \mu(d y) \mu(d x)\right)^{1 / p}\right)
\end{aligned}
$$

for all $u \in L^{p}$.

Under certain additional assumptions we can get rid of the $L^{p}$ norm on the right hand side.

2010 Mathematics Subject Classification: Primary 46E35; Secondary 46E30, 26D15, 60J75. Key words and phrases: Lipschitz space, Lorentz space, subcritical limiting embedding, domain of the Dirichlet form, stable process, Sobolev inequality, $d$-set, $h$-set, lower Ahlfors $d$-regularity. 
Corollary 2. Let $F \subset \mathbb{R}^{n}$ and $F=a F:=\{a x: x \in F\}$ for some $a>1$. Let $\mu$ be the d-dimensional Hausdorff measure and assume that it is $\sigma$-finite on $F$. Let $0<p<\infty$ and $0<\alpha<d / p$. There exists a constant $c=c\left(d, C_{1}, p, \alpha\right)$ such that

$$
\|u\|_{L^{p d /(d-\alpha p)}} \leq c\left(\iint_{F F} \frac{|u(x)-u(y)|^{p}}{|x-y|^{d+\alpha p}} \mu(d y) \mu(d x)\right)^{1 / p}
$$

for all $u \in L^{p}$.

The result applies e.g. if $F$ is a half-space in $\mathbb{R}^{n}$ (or more generally, an open cone) and $d=n$.

Inequality (2) for $p=2, \alpha<1$, and a $d$-set $F \subset \mathbb{R}^{n}$ was stated in [7, (2.3)] and applied in [7] to estimate the heat kernel of jump type processes (see also [4]). Such applications are our primary motivation to study such inequalities. They are also of interest in the study of function spaces on $d$-sets [17]. Furthermore, inequalities of this type have a close connection to Nash inequalities and heat kernel estimates (see [15, 8, 23, 1]).

Note that our proofs are different and more elementary than those in [7, 17. Interestingly, in our inequalities we allow for all $p>0$, rather than $p \geq 1, \alpha \in(0, d / p)$ may be larger than 1 , and we only assume lower Ahlfors $d$-regularity. Moreover, our methods yield an extension to Besov-Lipschitz spaces, given below.

We recall the definition of Lorentz spaces $L_{p, q}$ [17, 2]. We define the decreasing rearrangement $u^{*}$ of $u$ in the usual way,

$$
u^{*}(t)=\inf \{s: \mu(\{x:|u(x)|>s\}) \leq t\} .
$$

For $0<p, q<\infty$ we define

$$
\left\|u ; L_{p, q}\right\|=\left(\int_{0}^{\infty}\left(t^{1 / p} u^{*}(t)\right)^{q} \frac{d t}{t}\right)^{1 / q}, \quad\left\|u ; L_{p, \infty}\right\|=\sup _{t>0}\left(t^{1 / p} u^{*}(t)\right) .
$$

We say that $u \in L_{p, q}$ if $\left\|u ; L_{p, q}\right\|<\infty$.

For $0<p<\infty, 0<q \leq \infty$ and $\alpha>0$ we define the Besov-Lipschitz type space $\operatorname{Lip}_{0}(\alpha, p, q, F)=\left\{u \in L^{p}:\left\|u ; \operatorname{Lip}_{0}(\alpha, p, q, F)\right\|<\infty\right\}$, where

$$
\left\|u ; \operatorname{Lip}_{0}(\alpha, p, q, F)\right\|=\|u\|_{L^{p}}+\left\|\left(b_{\nu}\right)_{\nu=0}^{\infty}\right\|_{\ell^{q}},
$$

and the sequence $\left(b_{\nu}\right)_{\nu=0}^{\infty}$ is defined by

$$
b_{\nu}=2^{\nu \alpha}\left(2^{\nu d} \iint_{\rho(x, y)<2^{-\nu}}|u(x)-u(y)|^{p} \mu(d y) \mu(d x)\right)^{1 / p} .
$$

If $p, q \geq 1$, then (4) is a genuine norm.

The main result of this note is the following embedding theorem, which extends Proposition 6 in [17, p. 216]. 
Theorem 3. Let $F, \mu, \rho$ and $d$ be as in Theorem 1. Let $0<p<\infty$, $p \leq q \leq \infty$ and $0<\alpha<d / p$. Then there exists a constant $c=c\left(d, C_{1}, p, q, \alpha\right)$ such that for all $u \in \operatorname{Lip}_{0}(\alpha, p, q, F)$,

$$
\left\|u ; L_{p^{*}, q}\right\| \leq c\left\|u ; \operatorname{Lip}_{0}(\alpha, p, q, F)\right\|,
$$

where $p^{*}=p d /(d-\alpha p)$.

We may regard Theorem 3 as a subcritical case of a limiting embedding (see [22, Remark 11.5] for definitions and a further discussion).

We mention that the Hardy inequality of [12, 9, 3] is similar to (3), except that it estimates the weighted $L^{p}$ norm (and not $L^{p^{*}}$ ) by $\mathcal{E}$.

We note that the definition of $\operatorname{Lip}_{0}(\alpha, p, q, F)$ is very similar to the definition of the space $\Lambda_{p, q}^{d, \alpha}$ of Grigor'yan [13]. By the definition

$$
\left\|u ; \operatorname{Lip}_{0}(\alpha, p, q, F)\right\| \leq c\left\|u ; \Lambda_{p, q}^{d, \alpha}\right\|,
$$

and these two norms are equivalent for bounded $d$-sets $F$. Correspondingly, (6) holds with the norm $\operatorname{Lip}_{0}(\alpha, p, q, F)$ replaced by the norm of $\Lambda_{p, q}^{d, \alpha}$ in Theorem 3. See [13, 14] for a further discussion.

We now recall the definition of $\operatorname{Lip}(\alpha, p, q, F)$ of Jonsson and Wallin [17]. Assume that $F \subset \mathbb{R}^{n}$ and $\rho$ is the Euclidean distance. Let $\alpha>0$ and $k \in \mathbb{Z}$ satisfy $k<\alpha \leq k+1$. Let $\left\{f^{(j)}\right\}_{|j| \leq k}$ be a family of functions defined $\mu$-a.e. on $F$, where $j=\left(j_{1}, \ldots, j_{n}\right)$ is a multiindex and $|j|=j_{1}+\cdots+j_{n}$. We define $P_{j}$ and $R_{j}$ by requiring that

$$
P_{j}(x, y)=\sum_{|j+l| \leq k} \frac{f^{(j+l)}(y)}{l !}(x-y)^{l}, \quad x, y \in F,
$$

and that $f^{(j)}(x)=P_{j}(x, y)+R_{j}(x, y)$. The collection $\left\{f^{(j)}\right\}_{|j| \leq k}$ belongs to the Lipschitz space $\operatorname{Lip}(\alpha, p, q, F)$ if and only if $f^{(j)} \in L^{p}$ for $|j| \leq k$, and for $\nu=0,1,2, \ldots$ and $|j| \leq k$,

$$
\left(2^{\nu d} \iint_{|x-y|<2^{-\nu}}\left|R_{j}(x, y)\right|^{p} \mu(d x) \mu(d y)\right)^{1 / p} \leq 2^{-\nu(\alpha-|j|)} a_{\nu}
$$

for some sequence $\left(a_{\nu}\right) \in \ell^{q}$. The norm of $\left\{f^{(j)}\right\}_{|j| \leq k}$ in $\operatorname{Lip}(\alpha, p, q, F)$ is

$$
\sum_{|j| \leq k}\left\|f^{(j)}\right\|_{L^{p}}+\inf \left\|\left(a_{\nu}\right)\right\|_{\ell^{q}}
$$

where the infimum is taken over all possible sequences $\left(a_{\nu}\right)$. We see that the definition of $\operatorname{Lip}(\alpha, p, q, F)$ uses (a substitute of) Taylor expansion of $k$ th order, while $\operatorname{Lip}_{0}(\alpha, p, q, F)$ uses only increments of the function (0-order Taylor expansion). This motivates the notation $\operatorname{Lip}_{0}$.

For a function $f$ we put $\tilde{f}^{(0)}=f$ and $\tilde{f}^{(j)}=0$ if $|j|>0$. Clearly,

$$
\left\|f^{(0)} ; \operatorname{Lip}_{0}(\alpha, p, q, F)\right\|=\left\|\left\{\tilde{f}^{(j)}\right\} ; \operatorname{Lip}(\alpha, p, q, F)\right\| .
$$

In particular, we have $\operatorname{Lip}(\alpha, p, q, F)=\operatorname{Lip}_{0}(\alpha, p, q, F)$ for $\alpha \leq 1$. 
It seems that $\operatorname{Lip}_{0}(\alpha, p, q, F)$ is more appropriate to study jump processes on metric spaces (see [16, 20, 21]). For a $d$-set $F$ the $\operatorname{space}^{\operatorname{Lip}_{0}}\left(\alpha d_{w} / 4,2,2, F\right)$ is the domain of the Dirichlet form of a symmetric $\alpha$-stable process on $F$ 21], where $\alpha \in(0,2)$ and $d_{w}$ is the so-called walk dimension of $F$ [20]. Also, $\operatorname{Lip}_{0}\left(d_{w} / 2,2, \infty, F\right)$ is the domain of the Dirichlet form of the Brownian motion e.g. on the Sierpiński gasket $F \subset \mathbb{R}^{n}$, (see [16]). Our results shed light on domains of non-local Dirichlet forms defined on more general sets.

Notation $c=c(a, b, \ldots, z)$ means that the constant $0<c<\infty$ depends only on $a, b, \ldots, z$. All functions are assumed to be Borel measurable and complex-valued. In fact our results remain valid for Banach-space-valued functions $u$ (see (13), (14)).

2. Proof of Theorem 3 . In the following lemma we adopt the convention that $\frac{0}{0}=0$.

Lemma 4. For every $\varepsilon>0$,

$$
\sum_{n=1}^{\infty} a_{n} \leq a_{0}+3 \cdot 4^{\varepsilon} \sum_{n=1}^{\infty} \frac{a_{n}^{1+\varepsilon}}{\left(a_{n-1}+a_{n}+a_{n+1}\right)^{\varepsilon}}
$$

if $a_{n} \geq 0, n=0,1, \ldots$ and $a_{n}=0$ for large $n$.

Proof. Let

$$
A=\left\{n \in\{1,2, \ldots\}: a_{n} \geq \frac{1}{3}\left(a_{n-1}+a_{n+1}\right)\right\}, \quad B=\{1,2, \ldots\} \backslash A,
$$

and let $N$ be such that $B \subset\{1, \ldots, N\}$. For $n \in A$ we have $a_{n-1}+a_{n}+a_{n+1}$ $\leq 4 a_{n}$, hence

$$
\sum_{n \in A} a_{n} \leq 4^{\varepsilon} \sum_{n \in A} \frac{a_{n}^{1+\varepsilon}}{\left(a_{n-1}+a_{n}+a_{n+1}\right)^{\varepsilon}} .
$$

On the other hand, we have

$$
\sum_{n \in B} a_{n} \leq \frac{1}{3} \sum_{n \in B}\left(a_{n-1}+a_{n+1}\right) \leq \frac{1}{3} a_{0}+\frac{2}{3} \sum_{n=1}^{N} a_{n}+\frac{1}{3} a_{N+1},
$$

thus

$$
\frac{1}{3} \sum_{n \in B} a_{n}<\frac{1}{3} a_{0}+\frac{2}{3} \sum_{n \in A, n \leq N} a_{n}+\frac{1}{3} a_{N+1} .
$$

Since $N+1 \in A$, we obtain from $(12)$,

$$
\sum_{n \in B} a_{n}<a_{0}+2 \sum_{n \in A, n \leq N+1} a_{n}
$$

and this together with (11) completes the proof. 
REMARK 1 . We note that 10 does not hold for all sequences $a_{n} \geq 0$. Indeed, for $a_{n}=\exp \left(b^{n}\right)$, the right hand side of $(10)$ is finite if $b$ is large enough, while the left hand side is infinite. One can prove that 10 holds, with some constant $c=c(\varepsilon)$ instead of $3 \cdot 4^{\varepsilon}$ in (10), for all sequences $a_{n}=$ $o\left(q^{n}\right)$, where $q>0$; however, the proof is more complicated and will be omitted.

Proof of Theorem 3. Let $u \in \operatorname{Lip}_{0}(\alpha, p, q, F)$. Our goal is to prove (6) with $c$ independent of $u$. Note that

$$
\left\||u| ; L_{p^{*}, q}\right\|=\left\|u ; L_{p^{*}, q}\right\|
$$

and

$$
\left\||u| ; \operatorname{Lip}_{0}(\alpha, p, q, F)\right\| \leq\left\|u ; \operatorname{Lip}_{0}(\alpha, p, q, F)\right\|,
$$

hence it suffices to prove (6) for $u \geq 0$.

Furthermore, since for any $t>0$ we have

$$
\left\|u \wedge t ; \operatorname{Lip}_{0}(\alpha, p, q, F)\right\| \leq\left\|u ; \operatorname{Lip}_{0}(\alpha, p, q, F)\right\|,
$$

by the bounded convergence theorem we may also assume that $u$ is bounded. Finally, we may and will assume that $\|u\|_{L^{p}}=1$.

Let

$$
\begin{aligned}
& E_{n}=\left\{x \in F: u(x) \in\left[2^{n}, 2^{n+1}\right)\right\}, \\
& \mu_{n}=\mu\left(E_{n}\right), \quad n \in \mathbb{Z} .
\end{aligned}
$$

The idea of the proof is to estimate the norms in (6) by means of $\mu_{n}$ only, and then use special inequalities for sequences, including (10) and the Hardy inequality. While estimates for the $L^{p}$ and $L_{p^{*}, q}$ norms of $u$ by means of $\mu_{n}$ are straightforward, this is not the case for the $\ell^{q}$ norm of $\left(b_{\nu}\right)$. This is the place where the somewhat unusual terms $\mu_{n} /\left(\mu_{n-1}+\mu_{n}+\mu_{n+1}\right)$ arise, which result from considering $x$ and $y$ not in neighbouring sets $E_{n}$ (see (5) and (16)). We estimate the terms by using Lemma 4. The assumption $\|u\|_{L^{p}}=1$ implies that $\mu_{n-1}+\mu_{n}+\mu_{n+1} \leq 2^{-(n-1) p}$, thus $\mu_{n-1}+\mu_{n}+\mu_{n+1} \leq C_{1} / 2$ for $n \geq n_{0}=n_{0}\left(C_{1}, p\right)$.

We claim that for any $n \geq n_{0}$ there exists $\nu \in\{0,1,2, \ldots\}$ (depending on $n, u, \ldots$ ) such that

$$
\begin{aligned}
2^{n} \mu_{n}^{1 / p}\left(\mu_{n-1}\right. & \left.+\mu_{n}+\mu_{n+1}\right)^{-\alpha / d} \\
& \leq c 2^{\nu \alpha}\left(2^{\nu d} \int_{E_{n}} \int_{B\left(x, 2^{-\nu}\right)}|u(x)-u(y)|^{p} \mu(d y) \mu(d x)\right)^{1 / p}
\end{aligned}
$$

with constant $c=c\left(d, C_{1}, p, \alpha\right)$ independent of $n$. Here we adopt the convention that $0^{a}=0$ for $a<0$, hence the claim is obvious if $\mu_{n-1}+\mu_{n}+\mu_{n+1}=0$. 
We now prove the claim in the case when $\mu_{n-1}+\mu_{n}+\mu_{n+1}>0$. We have

$$
\begin{aligned}
b_{n, \nu} & :=\int_{E_{n}} \int_{B\left(x, 2^{-\nu}\right)}|u(x)-u(y)|^{p} \mu(d y) \mu(d x) \\
& \geq \int_{E_{n} B\left(x, 2^{-\nu}\right) \backslash\left(E_{n-1} \cup E_{n} \cup E_{n+1}\right)}|u(x)-u(y)|^{p} \mu(d y) \mu(d x) \\
& \geq 2^{(n-1) p} \mu_{n} \cdot \mu\left(B\left(x, 2^{-\nu}\right) \backslash\left(E_{n-1} \cup E_{n} \cup E_{n+1}\right)\right) \\
& \geq 2^{(n-1) p} \mu_{n}\left(C_{1} 2^{-\nu d}-\left(\mu_{n-1}+\mu_{n}+\mu_{n+1}\right)\right) .
\end{aligned}
$$

We take $\nu \in\{0,1,2, \ldots\}$ such that

$$
2\left(\mu_{n-1}+\mu_{n}+\mu_{n+1}\right) \leq C_{1} 2^{-\nu d}<2^{d+1}\left(\mu_{n-1}+\mu_{n}+\mu_{n+1}\right) .
$$

Then

$$
b_{n, \nu} \geq \frac{C_{1}}{2} 2^{(n-1) p} \mu_{n} 2^{-\nu d},
$$

hence

$$
2^{\nu \alpha}\left(2^{\nu d} b_{n, \nu}\right)^{1 / p} \geq c\left(d, C_{1}, p, \alpha\right)\left(\mu_{n-1}+\mu_{n}+\mu_{n+1}\right)^{-\alpha / d} 2^{n} \mu_{n}^{1 / p},
$$

and the claim is proven.

We will first prove (6) in the case when $q<\infty$. Observe that $2^{n} \leq$ $u^{*}(t)<2^{n+1}$ if $\sum_{k>n} \mu_{k}<t<\sum_{k \geq n} \mu_{k}$. Hence

$$
\begin{aligned}
\left\|u ; L_{p^{*}, q}\right\|^{q} & =\int_{0}^{\infty}\left(t^{1 / p^{*}} u^{*}(t)\right)^{q} \frac{d t}{t} \\
& \leq 2^{q} \sum_{n \in \mathbb{Z}} \int_{\sum_{k>n} \mu_{k}}^{\sum_{k \geq n} \mu_{k}} t^{q / p^{*}-1} 2^{n q} d t \\
& =\frac{2^{q} p^{*}}{q} \sum_{n \in \mathbb{Z}}\left(\left(\sum_{k \geq n} \mu_{k}\right)^{q / p^{*}}-\left(\sum_{k>n} \mu_{k}\right)^{q / p^{*}}\right) 2^{n q} \\
& \leq \frac{2^{q} p^{*}}{q} \sum_{n \in \mathbb{Z}}\left(\sum_{k \geq n} \mu_{k}\right)^{q / p^{*}} 2^{n q} .
\end{aligned}
$$

We use the following variant of the Hardy inequality ([17, Lemma 3, p. 121], [19]), valid for $s, q>0$ :

$$
\sum_{n=n_{0}}^{\infty}\left(\sum_{k \geq n} \mu_{k}\right)^{s} 2^{n q} \leq c\left(n_{0}, s, q\right) \sum_{n=n_{0}}^{\infty} \mu_{n}^{s} 2^{n q},
$$

and the estimate $\sum_{k \geq n} \mu_{k} \leq 2^{-n p}$, which follows from $\|u\|_{L^{p}}=1$. We deduce 
from 17 that

$$
\begin{aligned}
\left\|u ; L_{p^{*}, q}\right\|^{q} & \leq c \sum_{n<n_{0}} 2^{-n p q / p^{*}} 2^{n q}+c \sum_{n=n_{0}}^{\infty} \mu_{n}^{q / p^{*}} 2^{n q} \\
& \leq c\|u\|_{L_{p}}^{q}+c \sum_{n=n_{0}}^{\infty} \mu_{n}^{q / p^{*}} 2^{n q}
\end{aligned}
$$

where $c=c\left(d, C_{1}, p, q, \alpha\right)$.

Since $u$ is bounded, $\mu_{n}=0$ for all large $n$. We are going to apply Lemma 4 to $a_{n}=\mu_{n}^{\gamma} 2^{n q}$, where $\gamma=q / p^{*}$, and $\varepsilon=\alpha q /(\gamma d)>0$. Observe that $\gamma(1+\varepsilon)$ $=q / p$. Note that

$$
\frac{a_{n}^{1+\varepsilon}}{\left(a_{n-1}+a_{n}+a_{n+1}\right)^{\varepsilon}} \leq c \frac{\mu_{n}^{\gamma(1+\varepsilon)}}{\left(\mu_{n-1}+\mu_{n}+\mu_{n+1}\right)^{\gamma \varepsilon}} \cdot 2^{n q}
$$

with $c=c(d, p, q, \alpha)$. Thus by Lemma 4 and the inequality 15 raised to the $q$ th power we obtain

$$
\begin{aligned}
\sum_{n=n_{0}}^{\infty} 2^{n q} \mu_{n}^{\gamma} & \leq c \sum_{n=n_{0}}^{\infty} \frac{\mu_{n}^{q / p}}{\left(\mu_{n-1}+\mu_{n}+\mu_{n+1}\right)^{\alpha q / d}} 2^{n q}+2^{\left(n_{0}-1\right) q} \mu_{n_{0}-1}^{\gamma} \\
& \leq c \sum_{n=n_{0}}^{\infty} 2^{\nu(n) q \alpha}\left(2^{\nu(n) d} b_{n, \nu(n)}\right)^{q / p}+2^{\left(n_{0}-1\right) q} \mu_{n_{0}-1}^{\gamma} \\
& \leq c \sum_{n=n_{0}}^{\infty} \sum_{\nu=0}^{\infty} 2^{\nu q \alpha}\left(2^{\nu d} b_{n, \nu}\right)^{q / p}+c\|u\|_{L^{p}}^{q}
\end{aligned}
$$

with $c=c\left(d, C_{1}, p, q, \alpha\right)$. We note that in (19) above $\nu(n)$ depends also on $n$ and $u$, but the dependence vanishes in (20). The first term in 20 is now estimated as follows:

$$
\begin{aligned}
\sum_{n=n_{0}}^{\infty} \sum_{\nu=0}^{\infty} 2^{\nu q \alpha}\left(2^{\nu d} b_{n, \nu}\right)^{q / p} & \\
& =\sum_{\nu=0}^{\infty} 2^{\nu q \alpha} \sum_{n=n_{0}}^{\infty}\left(2^{\nu d} b_{n, \nu}\right)^{q / p} \leq \sum_{\nu=0}^{\infty} 2^{\nu q \alpha}\left(2^{\nu d} \sum_{n=n_{0}}^{\infty} b_{n, \nu}\right)^{q / p} \\
& \leq \sum_{\nu=0}^{\infty} 2^{\nu q \alpha}\left(2^{\nu d} \iint_{\rho(x, y)<2^{-\nu}}|u(x)-u(y)|^{p} \mu(d y) \mu(d x)\right)^{q / p} \\
& \leq\left\|u ; \operatorname{Lip}_{0}(\alpha, p, q, F)\right\|^{q}
\end{aligned}
$$

Putting (18), 20) and (21) together we obtain (6). 
It remains to show (6) in the case when $q=\infty$. We have

$$
\left\|u ; L_{p^{*}, \infty}\right\| \leq 2 \sup _{n}\left(\sum_{k \geq n} \mu_{k}\right)^{1 / p^{*}} 2^{n} .
$$

Observe that for $n \leq n_{0}$,

$$
\left(\sum_{k \geq n} \mu_{k}\right)^{1 / p^{*}} 2^{n} \leq\left(2^{-n p}\right)^{1 / p^{*}} 2^{n} \leq 2^{n_{0}\left(1-p / p^{*}\right)},
$$

hence

$$
\sup _{n \leq n_{0}}\left(\sum_{k \geq n} \mu_{k}\right)^{1 / p^{*}} 2^{n} \leq c\left(d, C_{1}, p, \alpha\right)\|u\|_{L^{p}}
$$

Now let

$$
S=\sup _{n \geq n_{0}}\left(\sum_{k \geq n} \mu_{k}\right)^{1 / p^{*}} 2^{n} .
$$

We have $S<\infty$, because $u$ is bounded. Let $N \geq n_{0}$ be such that

$$
\left(\sum_{k \geq N} \mu_{k}\right)^{1 / p^{*}} 2^{N} \geq \frac{3}{4} S
$$

If $N=n_{0}$, then $S \leq c\left(d, C_{1}, p, \alpha\right)\|u\|_{L^{p}}$ by 22). Henceforth we assume that $N>n_{0}$. By (15) we get

$$
\sup _{n \geq n_{0}} 2^{n} \mu_{n}^{1 / p}\left(\mu_{n-1}+\mu_{n}+\mu_{n+1}\right)^{-\alpha / d} \leq c\left\|u ; \operatorname{Lip}_{0}(\alpha, p, \infty, F)\right\|
$$

(see (5)). From (23) and the inequalities $\left(\sum_{k \geq n} \mu_{k}\right)^{1 / p^{*}} 2^{n} \leq S$ for $n=N-1$ and $n=N+1$, we obtain, respectively,

$$
\begin{aligned}
\mu_{N-1}+\mu_{N}+\mu_{N+1} & \leq \sum_{k \geq N-1} \mu_{k} \leq\left(\frac{8}{3}\right)^{p^{*}} \sum_{k \geq N} \mu_{k}, \\
\mu_{N} & \geq\left(\left(\frac{3}{2}\right)^{p^{*}}-1\right) \sum_{k \geq N+1} \mu_{k} .
\end{aligned}
$$

Thus $\sum_{k \geq N} \mu_{k} \leq c\left(p^{*}\right) \mu_{N}$, hence by $1 / p^{*}=-\alpha / d+1 / p$ and (24),

$$
\begin{aligned}
\frac{3}{4} S & \leq\left(\sum_{k \geq N} \mu_{k}\right)^{1 / p^{*}} 2^{N} \leq c\left(\mu_{N-1}+\mu_{N}+\mu_{N+1}\right)^{-\alpha / d} 2^{N} \mu_{N}^{1 / p} \\
& \leq c\left\|u ; \operatorname{Lip}_{0}(\alpha, p, \infty, F)\right\| . \text { - }
\end{aligned}
$$

Proof of Theorem 1. By Theorem 3 applied to $p=q<\infty$ we have

$$
\begin{aligned}
\|u\|_{L_{p^{*}, p}} & \leq c\left\|u ; \operatorname{Lip}_{0}(\alpha, p, p, F)\right\| \\
& \leq c\left(\|u\|_{L^{p}}+\left(\iint_{\rho(x, y)<1} \frac{|u(x)-u(y)|^{p}}{\rho(x, y)^{d+\alpha p}} \mu(d y) \mu(d x)\right)^{1 / p}\right),
\end{aligned}
$$


and the theorem follows from the embedding $L_{p^{*}, p} \subset L^{p^{*}}$ for $p<p^{*}[2$, Proposition 4.2, p. 217].

Proof of Corollary 2. Denote $u^{(a)}(x)=u(a x)$ and

$$
\mathcal{E}(u)=\iint_{F F} \frac{|u(x)-u(y)|^{p}}{|x-y|^{d+\alpha p}} \mu(d y) \mu(d x) .
$$

It is easy to check that $\left\|u^{(a)}\right\|_{L^{s}}=a^{-d / s}\|u\|_{L^{s}}$ and $\mathcal{E}\left(u^{(a)}\right)=a^{-d+\alpha p} \mathcal{E}(u)$. Hence by (2) applied to $u^{\left(a^{n}\right)}$ we obtain

$$
\|u\|_{L^{p d /(d-\alpha p)}} \leq c\left(a^{-n \alpha}\|u\|_{L^{p}}+\mathcal{E}(u)^{1 / p}\right)
$$

and the corollary follows by letting $n \rightarrow \infty$.

Note. One can simplify the proof of Corollary 2 to get a stronger result. Namely, assume instead of (1) that for some $C_{1}, d, r_{0}>0$,

$$
\mu(B(x, r)) \geq C_{1} r^{d} \quad \text { for all } 0<r \leq r_{0} \text { and } x \in F .
$$

Then the new measure $\tilde{\mu}(A):=\mu(A) r_{0}^{-d}$ and the new metric $\tilde{\rho}(x, y):=$ $\rho(x, y) / r_{0}$ satisfy (1), hence (2) holds. Coming back to $\mu$ and $\rho$ we get the following corollary.

Corollary 5. Assume that (25) holds. If $0<p<\infty$ and $0<\alpha<d / p$, then there exists a constant $c=c\left(d, C_{1}, p, \alpha\right)$ such that

$$
\begin{aligned}
& \|u\|_{L^{p d /(d-\alpha p)}} \\
& \quad \leq c\left(r_{0}^{-\alpha}\|u\|_{L^{p}}+\left(\iint_{\rho(x, y)<r_{0}} \frac{|u(x)-u(y)|^{p}}{\rho(x, y)^{d+\alpha p}} \mu(d y) \mu(d x)\right)^{1 / p}\right)
\end{aligned}
$$

for all $u \in L^{p}$. In particular, if (25) holds for all $r_{0}>0$, then

$$
\|u\|_{L^{p d /(d-\alpha p)}} \leq c\left(\iint_{F F} \frac{|u(x)-u(y)|^{p}}{\rho(x, y)^{d+\alpha p}} \mu(d y) \mu(d x)\right)^{1 / p}
$$

Acknowledgements. The author wishes to thank Krzysztof Bogdan, Andrzej Stós, and Paweł Sztonyk for helpful discussions, and the anonymous referee for the valuable comments.

This research was partially supported by KBN 1 P03A 02629.

\section{References}

[1] D. Bakry, T. Coulhon, M. Ledoux, and L. Saloff-Coste, Sobolev inequalities in disguise, Indiana Univ. Math. J. 44 (1995), 1033-1074.

[2] C. Bennett and R. Sharpley, Interpolation of Operators, Pure Appl. Math. 129, Academic Press, Boston, MA, 1988.

[3] K. Bogdan and B. Dyda, The best constant in a fractional Hardy inequality, Math. Nachr., to appear. 
[4] K. Bogdan, A. Stós, and P. Sztonyk, Harnack inequality for stable processes on d-sets, Studia Math. 158 (2003), 163-198.

[5] M. Bricchi, Complements and results on h-sets, in: Function Spaces, Differential Operators and Nonlinear Analysis (Teistungen, 2001), Birkhäuser, Basel, 2003, 219229.

[6] -, Tailored Besov spaces and h-sets, Math. Nachr. 263/264 (2004), 36-52.

[7] Z.-Q. Chen and T. Kumagai, Heat kernel estimates for stable-like processes on d-sets, Stochastic Process. Appl. 108 (2003), 27-62.

[8] -, Heat kernel estimates for jump processes of mixed types on metric measure spaces, Probab. Theory Related Fields 140 (2008), 277-317.

[9] B. Dyda, A fractional order Hardy inequality, Illinois J. Math. 48 (2004), 575-588.

[10] D. Edmunds and H. Triebel, Spectral theory for isotropic fractal drums, C. R. Acad. Sci. Paris Sér. I Math. 326 (1998), 1269-1274.

[11] - Eigenfrequencies of isotropic fractal drums, in: The Maz'ya Anniversary Collection, Vol. 2 (Rostock, 1998), Oper. Theory Adv. Appl. 110, Birkhäuser, Basel, 1999, 81-102.

[12] R. Frank, E. Lieb, and R. Seiringer, Hardy-Lieb-Thirring inequalities for fractional Schrödinger operators, J. Amer. Math. Soc. 21 (2008), 925-950.

[13] A. Grigor'yan, Heat kernels and function theory on metric measure spaces, in: Heat Kernels and Analysis on Manifolds, Graphs, and Metric Spaces (Paris, 2002), Contemp. Math. 338, Amer. Math. Soc., Providence, RI, 2003, 143-172.

[14] A. Grigor'yan, J. Hu, and K.-S. Lau, Heat kernels on metric measure spaces and an application to semilinear elliptic equations, Trans. Amer. Math. Soc. 355 (2003), 2065-2095.

[15] J. Hu and T. Kumagai, Nash-type inequalities and heat kernels for non-local Dirichlet forms, Kyushu J. Math. 60 (2006), 245-265.

[16] A. Jonsson, Brownian motion on fractals and function spaces, Math. Z. 222 (1996), 495-504.

[17] A. Jonsson and H. Wallin, Function Spaces on Subsets of $\mathbf{R}^{n}$, Math. Rep. 2 (1984), xiv $+221 \mathrm{pp}$.

[18] V. Knopova and M. Zähle, Spaces of generalized smoothness on h-sets and related Dirichlet forms, Studia Math. 174 (2006), 277-308.

[19] L. Leindler, Generalization of inequalities of Hardy and Littlewood, Acta Sci. Math. (Szeged) 31 (1970), 279-285.

[20] K. Pietruska-Pałuba, On function spaces related to fractional diffusions on d-sets, Stoch. Stoch. Rep. 70 (2000), 153-164.

[21] A. Stós, Symmetric $\alpha$-stable processes on d-sets, Bull. Polish Acad. Sci. Math. 48 (2000), 237-245.

[22] H. Triebel, The Structure of Functions, Monogr. Math. 97, Birkhäuser, Basel, 2001.

[23] N. T. Varopoulos, Hardy-Littlewood theory for semigroups, J. Funct. Anal. 63 (1985), 240-260.

Bartłomiej Dyda

Institute of Mathematics and Computer Science

Wrocław University of Technology

Wybrzeże Wyspiańskiego 27

50-370 Wrocław, Poland

E-mail: bdyda@pwr.wroc.pl 